\title{
Curvas de Regularização para Reservatórios Parcialmente Cheios com Confiabilidade Constante
}

\author{
Heinz Dieter O. A. Fill e Kátia Luciane Neira \\ Universidade Federal do Paraná \\ heinzfill@yahoo.com; katia@pab.eng.br
}

Recebido: 01/12/06 - revisado: 03/09/07 - aceito: 05/12/07

RESUMO

Este estudo visa estabelecer a capacidade de atendimento de um sistema elétrico interligado constituído exclusivamente de usinas hidrelétricas em função do estado inicial do reservatório de energia. Uma política de atendimento razoável é manter constante o risco de falha futura do sistema considerando a cada momento o estado do reservatório de regularização. Isso evidentemente implica em uma redução da geração hidrelétrica quando o estado inicial dos reservatórios está abaixo do armazenamento máximo. Essa redução pode ser suprida por usinas térmicas complementares, porém na impossibilidade do uso desta fonte de geração implicará em racionamento. É proposto um método para avaliar essa redução por meio de simulações de Monte Carlo usando séries sintéticas de energias naturais afluentes. Para a geração das séries sintéticas analisaramse dois cenários distintos, um utilizando estatísticas da série histórica completa, de 1931-1995 e outro usando uma sub-série de 1931-1980 e que resultou em um coeficiente de autocorrelação mais coerente com estudos energéticos anteriores. A comparação entre os resultados da série histórica e das séries sintéticas mostra que a energia firme histórica corresponde a um tempo de retorno de aproximadamente 100 anos para a série completa e cerca de 70 anos para a sub-série 1931-1980. Conclui-se que a avaliação da redução da energia garantida para o reservatório de energia parcialmente cheio é viável e que o método pode ser utilizado na prática para estabelecer critérios de operação térmica e racionamento preventivo do sistema hidrotérmico brasileiro. Analisou-se também a determinação da redução da energia firme em função do armazenamento inicial ao simular o sistema ao longo do período histórico. Neste caso a redução, além do valor do armazenamento inicial, depende também do ano do histórico no qual se inicia a simulação. Este ponto coloca a questão de que na prática, a decisão sobre o valor da redução da geração hidráulica deve considerar se possível, além do estado do reservatório, o cenário futuro das afluências.

Palavras-chave: Método de Monte Carlo, séries sintéticas de energias, método da energia natural, curva de regularização, planejamento de racionamento.

\section{INTRODUÇÃO}

A falta de investimento no setor elétrico nas duas últimas décadas tem sido um dos grandes entraves ao crescimento econômico brasileiro, que fica refém das adversidades hidrológicas e de falhas no planejamento do sistema. Déficits no sistema elétrico, como o ocorrido em 2001, causam grandes prejuízos à economia e desaceleram o crescimento do país.

Portanto, é de grande interesse para um país voltado ao desenvolvimento encontrar meios de minimizar os riscos de novas crises energéticas.

Diversos países, como Inglaterra, Estados Unidos e Nova Zelândia, procurando tornar seus sistemas elétricos mais confiáveis, iniciaram reformas em seus modelos regulatórios no início da década de 90 .
O Brasil, seguindo estes passos, procurou reformular o setor elétrico em meados dos anos 90, introduzindo reformas profundas com privatizações e a tentativa de implantar uma política de incentivos à competição. Entretanto, devido aos ganhos decorrentes da diversidade hidrológica verificou-se a necessidade de preservar os benefícios da operação integrada e otimizada do sistema. Essa necessidade levou à criação do Mecanismo de Realocação de Energia - MRE.

O MRE é um mecanismo de compartilhamento do risco hidrológico e de rateio do retorno financeiro associado à otimização do sistema hidrotérmico. Essa otimização é realizada através de um despacho centralizado pelo Operador Nacional do Sistema - ONS.

Entretanto, mesmo encontrando estímulos que incentivem os investimentos no setor, continua sendo primordial a prevenção de novos colapsos 
energéticos, através de medidas tomadas na operação do sistema que minimizem os riscos de falta de energia.

Com este objetivo, tem sido proposta a operação dos reservatórios dos aproveitamentos hidrelétricos do sistema interligado, mantendo o risco do seu esvaziamento completo sempre abaixo de um valor limite.

$\mathrm{Na}$ iminência da violação desse princípio deve-se iniciar um racionamento energético preventivo controlado e com intensidade menor, causando um impacto inferior em todos os setores da sociedade, comparado à situação descontrolada resultante do esvaziamento total dos reservatórios.

A análise do desempenho de um sistema hidrelétrico interligado pode ser efetuada através do chamado método da energia natural (CANAMBRA, 1969). FILL (1980) comparando esse método ao resultado de simulações detalhadas concluiu que o erro não ultrapassa 5\%, o que devido às incertezas envolvendo dados básicos pode ser considerado aceitável.

O método da energia natural apresenta um enfoque bastante simplificado para a simulação de um sistema hidrelétrico, mas conduz a excelentes resultados globais, principalmente se as várias usinas que compõem o sistema são dotadas de boa regularização e as interconexões elétricas entre usinas não apresentam limitações importantes. Suas limitações dizem respeito a locais moderadamente regularizados hidraulicamente (Canambra, 1969).

O método da energia natural (Natural $\boldsymbol{E}$ nergy Hydrograph) também conhecido como método do reservatório equivalente (Kelman e Pereira, 1977) consiste em transformar as vazões afluentes a cada usina em energias afluentes (MWméd) usando para tanto uma produtividade média e a seguir somando as energias afluentes obtendo a chamada energia natural do sistema. A água armazenada em vários reservatórios é transformada em energia armazenada (MWmês) e também somada para todo sistema. A seguir simula-se a operação do sistema como sendo a operação de uma única usina com um único reservatório. Alguns refinamentos são ainda possíveis considerando a energia natural controlável (afluente aos reservatórios) e conservando a energia natural pela potência instalada nas usinas a fio de água sem regularização a montante.

Assim um sistema totalmente interligado e com capacidade de regularização bem distribuída apresenta-se similar a um reservatório único que deve regularizar retiradas de água, e onde o conceito da curva de regularização permite determinar a vazão garantida em função do volume útil disponível e do grau de confiabilidade.

A utilização de uma curva de regularização, para um dado risco e volume útil máximo, considerando como volume útil inicial um estado de enchimento parcial do reservatório, permite estimar qual redução da vazão regularizada, à medida que o reservatório seja esvaziado, é necessário para manter o risco de falha constante. Essa curva pode ser obtida através de simulações de Monte Carlo ou pela teoria estocástica dos reservatórios.

Essa idéia pode ser aplicada igualmente à operação de um sistema hidrelétrico. Neste caso, reduz-se a parcela da carga atendida pelas hidrelétricas (ao custo de um aumento na geração termelétrica ou em situações extremas por racionamento) à medida que a energia armazenada no sistema diminui, procurando-se sempre manter constante a confiabilidade futura do sistema, condicionada ao estado atual dos reservatórios.

$\mathrm{Na}$ operação de um sistema hidrelétrico, dado o caráter aleatório das vazões naturais e o tamanho finito dos reservatórios existe sempre um risco de não atendimento da carga, o que na prática leva a possíveis racionamentos e cortes de fornecimento. Isto não significa que um sistema termelétrico seja imune a riscos de racionamento, mas apenas que a natureza e, por conseguinte a formulação matemática da análise do risco seja diferente. Em geral evita-se um racionamento extremamente severo e incontrolado, o que ocorreria no caso de deplecionar completamente os reservatórios de todo sistema. Coloca-se então a questão de quando e em que proporção, deve-se iniciar o racionamento.

Uma idéia razoável e consistente com a aversão ao risco proposta por Gomide (2002) é procurar manter o risco do esvaziamento completo dos reservatórios sempre abaixo de um valor limite préfixado. Essa idéia tem como conseqüência lógica o estabelecimento de regras de operação, que em função do armazenamento inicial disponível, definem a energia que pode ser atendida com esse risco para um dado horizonte de planejamento.

Um dos problemas fundamentais no planejamento da expansão de um sistema hidrelétrico é a avaliação do seu desempenho no atendimento do mercado sob condições hidrológicas adversas. Esse desempenho, que pode ser quantificado por um conjunto de índices ou grandezas, freqüentemente conflitantes entre si, evidentemente dependerá das regras operativas do sistema. Entre essas regras, é importante a definição de uma política de racionamento preventivo, cujo propósito é, em síntese, trocar déficits grandes embora pouco prováveis, por 
déficits pequenos, porém mais freqüentes (Fill e Groszewicz, 1987). Essa idéia vem ao encontro à tendência atual em evitar sistemas onde as conseqüências de uma falha, embora pouco provável, produzem efeitos extremamente adversos, ou seja, falhas com segurança vs. segurança contra falhas (Hashimoto et al., 1982).

Para que todos os agentes do setor elétrico e também os consumidores possam entender claramente as razões de um eventual racionamento é necessário que as regras e os critérios que norteiam a imposição de cortes de energia sejam simples e transparentes, podendo ser entendidos não só por especialistas em pesquisa operacional. $\mathrm{O}$ presente estudo buscou justamente contribuir neste particular desenvolvendo um critério objetivo (manutenção do nível de confiabilidade) e as regras daí decorrentes (curvas-guia de operação) para o setor elétrico e em particular para o sistema interligado Sul-Sudeste.

Deve ficar claro que o propósito principal deste estudo não é apresentar uma regra operativa acabada e operacional para o setor elétrico, mas propor um método para definir tal regra e demonstrar a viabilidade de sua operacionalização. Certamente existem condicionantes adicionais que fogem ao conhecimento dos autores e que precisam ser incorporados ao método.

Além disso, como será mostrado, a série de energias afluentes disponível para este trabalho aparentemente apresenta algumas inconsistências que precisam ser investigadas com maior profundidade e corrigidas, o que não foi feito pela dificuldade de acesso aos dados originais do ONS. Entretanto essa limitação não invalida o método proposto.

\section{METODOLOGIA}

O presente estudo utilizou para aplicação do método analisado, o subsistema integrado SulSudeste do Brasil, configuração 2001. O Centro de Hidráulica de Hidrologia Prof. Parigot de Souza CEHPAR cedeu gentilmente a série de energias afluentes deste sistema para o período 1931 a 1996 (Kaviski, 2002).

O valor da energia máxima armazenada $\left(\mathrm{S}_{\text {máx }}\right)$ para o subsistema Sul-Sudeste segundo Kaviski (2002) correspondente à configuração de 2001 é 173.551 MWmês e o valor da energia firme do sistema é igual a 26.789 MWmédios.

Os dados fornecidos foram reorganizados com base no ano hidrológico, para o cálculo dos parâmetros estatísticos amostrais. $\mathrm{O}$ ano hidrológico inicia em maio e conclui-se em abril do ano seguinte.

Uma nova série histórica, com 65 anos, cada um iniciando em maio do ano "i" e término em abril do ano " $i+1$ " $i=(1931,1932, \ldots, 1995)$ foi formada. Calcularam-se as afluências médias anuais e determinaram-se as suas estatísticas relevantes para a geração de séries sintéticas. A média encontrada foi igual a 33.104 MW ano/ano, o desvio padrão 7.053 MW ano/ano, o coeficiente de assimetria 1,13 e coeficiente autocorrelação 0,54.

Uma das estatísticas que mais chamou atenção nesta análise, foi o coeficiente de autocorrelação em série de 0,54, muito superior ao esperado. Gomide (1986) menciona que na região Sudeste do Brasil é comum encontrar coeficientes de autocorrelação na faixa de $15 \%$ a 25\%. Kelman (1987) obteve para uma estação fluviométrica típica (Rio Paraíba do Sul em Barra do Piraí) um coeficiente de autocorrelação de 26\%. Fill (1989) adotou um valor de $30 \%$ ao propor um método analítico de avaliação da energia garantida. CEHPAR (1987) analisando a série de vazões do rio Paraná em Guaíra obteve $18,3 \%$. Assim o valor de $54 \%$ encontrado na série de energias afluentes parece definitivamente exagerado.

Analisando o comportamento dos coeficientes de autocorrelação através de médias móveis, conforme apresentado na figura 1 observa-se que os coeficientes de autocorrelação aumentaram muito quando anos posteriores a 1983 (a partir da série \#34) foram incluídos na amostra.

Optou-se então analisar o método proposto não só através da série histórica de energia natural do Sistema Sul-Sudeste na configuração 2001 fornecida pelo CEHPAR (Kaviski, 2002) com período de 1931 a 1995, mas também através de uma série histórica reduzida, com 50 anos, iniciando em maio do ano 1931 e término em abril do ano 1981, que apresenta um coeficiente de autocorrelação mais coerente com outros estudos.

O período de maio de 1931 a abril de 1981 utilizado para a série histórica alternativa possui média 31.282 MW ano/ano, desvio padrão 5.493 MW ano/ano, coeficiente de assimetria 0,27 e coeficiente autocorrelação igual a 0,33. Em função da definição do ano hidrológico este período será denominado 1931-1980.

Esses valores são similares aos usados por vários pesquisadores em estudos anteriores (Gomide, 1986; Kelman, 1987; Fill, 1989 e CEHPAR, 1987), além de estar próximo à média das sub-amostras de 20 anos $(\hat{r}=0,34)$. 


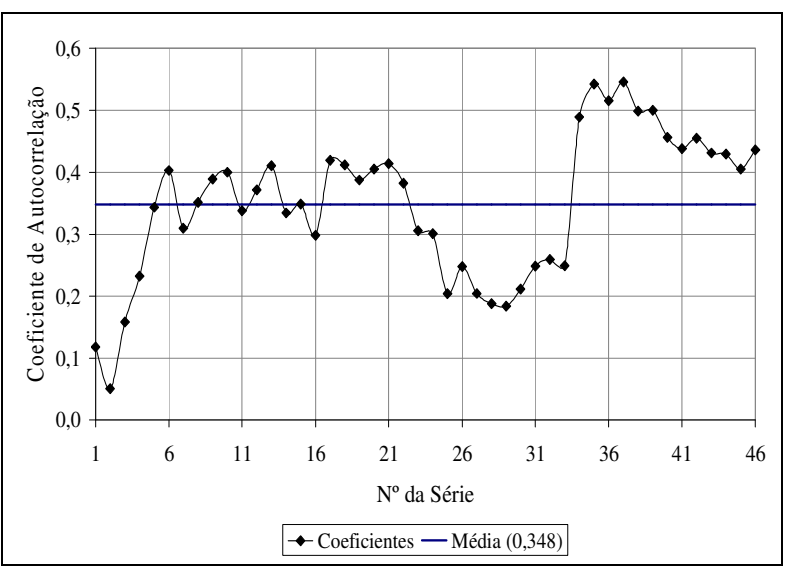

Figura 1 - Médias móveis para intervalo de 20 anos - coeficientes de autocorrelação anual da série histórica de energias afluentes do sistema sul-sudeste

\section{Geração de Séries Sintéticas Anuais}

A geração de séries sintéticas anuais foi realizada a partir da formulação de um modelo computacional desenvolvido na linguagem Turbo pascal for Windows, versão 7.0.

A geração de variáveis aleatórias normalmente distribuídas baseou-se em um modelo apresentado por Press et al. (1989) gerando inicialmente variáveis uniformes e usando o método de BoxMuller (Press et al., 1989) para transformar variáveis aleatórias uniformemente distribuídas, $U_{t}$, em variáveis aleatórias normais padrão, $Z_{t}$.

Para obter séries com distribuição LN3 recorre-se a transformação de Thomas-Fiering (Kelman, 1987).

$x_{t}=\xi+\exp \left(Z_{t} \sigma_{y}+\mu_{y}\right)$

Sendo:

$x_{t}=$ energia afluente no mês $t$ e distribuída LN3;

$\xi=$ limite inferior das energias afluentes.

$\mu_{y}=E\left[\ln \left(X_{t}-\xi\right)\right]$ e $\sigma_{y}^{2}=\operatorname{Var}\left[\ln \left(X_{t}-\xi\right)\right]$

Os parâmetros $\sigma_{y}, \mu_{y}$ e $\xi$ são estimados a partir da amostra pelo método dos quantis combinado com o método da máxima verossimilhança (Stedinger, 1980) mediante as equações:

$$
\begin{aligned}
& \hat{\xi}=\frac{x_{(1)} x_{(n)}-x_{\text {mediana }}^{2}}{x_{(1)}+x_{(n)}-2 x_{\text {mediana }}}, \hat{\mu}_{y}=\frac{\sum_{1}^{n} \ln \left(x_{i}-\xi\right)}{n} \mathrm{e} \\
& \hat{\sigma}_{y}^{2}=\frac{\sum_{1}^{n}\left[\ln \left(x_{i}-\xi\right)-\mu_{y}\right]^{2}}{n}
\end{aligned}
$$

Onde:

$x_{(i)}=$ i-ésimo valor ordenado da energia média anual da série histórica;

$x_{\text {médiana }}=$ mediana dos valores observados da energia média anual;

$n$ = número de observações na série histórica.

Mediante este algoritmo foram geradas 1.000 séries sintéticas de energias afluentes anuais com 50 anos de extensão cada uma.

\section{Desagregação das Séries Anuais em Mensais}

Como o intervalo de discretização das séries deve ser mensal, foi utilizado o modelo de desagregação através de cenários hidrológicos, proposto por Groszewicz et al. (1991). Este método de desagregação foi comparado por Groszewicz et al. (1991) com outros métodos clássicos como o método de Valência e Schaake descrito por Kelman (1987) apresentando resultados equivalentes.

Foi calculada para cada ano da série histórica a razão entre as energias naturais médias mensais e a média anual obtendo-se então 65 cenários de coeficientes de desagregação cada um constituído por um vetor com 12 elementos.

A partir da série sintética de energia natural anual gerada, sorteou-se para cada ano um desses cenários e obtiveram-se as energias naturais mensais. Após o sorteio foi verificada a compatibilidade da energia natural do $12^{\circ}$ mês do ano $i$ com o $1^{\circ}$ mês do ano $i+1$ Considerou-se satisfatória a compatibilidade entre o $12^{\circ}$ mês do ano $i$ com o $1^{\circ}$ mês do ano $i+1, \operatorname{com} i=(1,2, \ldots, 64)$ se a razão entre as energias naturais correspondentes se situava entre o máximo e o mínimo desta mesma razão na série histórica. No caso de incompatibilidade, efetuou-se novo sorteio.

\section{Verificação e validação do modelo}

Segundo Kelman (1987), a confiança que se possa ter em um modelo estocástico de vazões de 
Tabela 1 - Comparação de valores populacionais e amostrais dos parâmetros das séries geradas (1931-1995)

\begin{tabular}{|c|c|c|c|c|c|c|}
\hline \multirow{3}{*}{$\begin{array}{l}\text { PARÂMETRO } \\
(\theta)\end{array}$} & \multicolumn{3}{|c|}{ ESPAÇO REAL } & \multicolumn{3}{|c|}{ ESPAÇO LOGARÍTMICO } \\
\hline & Populacional & \multicolumn{2}{|l|}{ Amostral } & \multirow{2}{*}{$\begin{array}{l}\text { Populacional } \\
\theta\end{array}$} & \multicolumn{2}{|l|}{ Amostral } \\
\hline & $\theta$ & $E(\theta)$ & $\operatorname{Var}(\theta)$ & & $E(\theta)$ & $\operatorname{Var}(\theta)$ \\
\hline MÉDIA & 33.150 & 33.077 & 3.018 .815 & 9,704 & 9,699 & 0,009 \\
\hline VARIÂNCIA & 54.227 .682 & 52.315 .333 & $4,29 \mathrm{E}+14$ & 0,159 & 0,155 & 0,001 \\
\hline $\begin{array}{c}\text { AUTOCORRELA- } \\
\text { ÇÃO }\end{array}$ & 0,541 & 0,407 & 0,016 & 0,485 & 0,441 & 0,014 \\
\hline DESVIO PADRÃO & 7.364 & 7.101 & 1.891.216 & 0,399 & 0,391 & 0,002 \\
\hline
\end{tabular}

Tabela 2 - Comparação de valores populacionais e amostrais dos parâmetros das séries geradas (1931-1980)

\begin{tabular}{|c|c|c|c|c|c|c|}
\hline \multirow{3}{*}{ 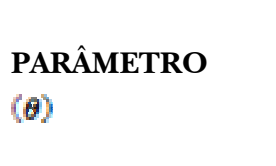 } & \multicolumn{3}{|c|}{ ESPAÇO REAL } & \multicolumn{3}{|c|}{ ESPAÇO LOGARÍTMICO } \\
\hline & \multirow{2}{*}{$\begin{array}{l}\text { Populacional } \\
\theta\end{array}$} & \multicolumn{2}{|l|}{ Amostral } & \multirow{2}{*}{$\begin{array}{l}\text { Populacional } \\
\theta\end{array}$} & \multicolumn{2}{|l|}{ Amostral } \\
\hline & & $E(\theta)$ & $\operatorname{Var}(\theta)$ & & $E(\theta)$ & $\operatorname{Var}(\theta)$ \\
\hline MÉDIA & 31.310 & 31.230 & 1.214 .572 & 10,283 & 10,280 & 0,001 \\
\hline VARIÂNCIA & 31.520 .347 & 30.391 .669 & $6,01 \mathrm{E}+13$ & 0,035 & 0,034 & 0,0001 \\
\hline $\begin{array}{c}\text { AUTOCORRELA- } \\
\text { CÃO }\end{array}$ & 0,332 & 0,279 & 0,018 & 0,330 & 0,286 & 0,017 \\
\hline DESVIO PADRÃO & 5.614 & 5.470 & 474.362 & 0,186 & 0,183 & 0,0004 \\
\hline
\end{tabular}

pende da capacidade que ele tenha de preservar, nas séries sintéticas, algumas propriedades observadas na série histórica. Diz-se que um modelo preserva uma determinada propriedade quando não se pode distinguir estatisticamente a série histórica da sintética, com base nas observações desta propriedade nas duas séries.

De acordo com Kelman e Pereira (1977) e Stedinger e Taylor (1982), quando alguma propriedade é utilizada para a determinação de um parâmetro de modelo, esta propriedade é automaticamente preservada, por construção. Nesse caso, comparar a observação da propriedade da série sintética com seu correspondente da série histórica serve apenas para verificar a adequação do programa de computador utilizado, e não para validar o modelo. A adequação do modelo foi analisada sob dois aspectos:

a) reprodução dos parâmetros básicos utilizados na geração das séries e; b) similaridade com a série histórica no que tange a características relevantes para a definição de curvas de regularização.

O primeiro aspecto essencialmente serve para confirmar que o modelo de geração esteja corretamente programado, ou seja, que reproduza as estatísticas que é suposto reproduzir. Os parâmetros básicos usados na geração das séries sintéticas na seqüência denominados parâmetros populacionais são a média, o desvio padrão e o coeficiente de autocorrelação tanto no espaço real como no espaço logarítmico, além do parâmetro locacional ( $\xi=$ 15.412) da distribuição LN3 que não foi testado.

A tabela 1 compara, para o caso da série 1931-1996, os valores populacionais (usados na geração das series sintéticas) com os valores amostrais obtidos a partir das 1.000 séries de afluências geradas. A tabela 2 apresenta a mesma comparação para a série 1931-1980. Os valores populacionais diferem ligeiramente dos valores da série histórica devido ao 
método de estimação dos parâmetros da distribuição LN3 usado. A diferença entre os valores populacionais e amostrais são aceitáveis quando comparados com as expressões teóricas publicadas por Kendal (1952) e Loucks et al (1981) para a variância amostral da média e variância de séries autocorrelacionadas.

Observa-se que os valores amostrais são bastante próximos dos valores teóricos o que valida o modelo de geração, isto é, que as diferenças entre os valores teóricos e observados podem ser atribuídos ao acaso.

Quanto ao segundo aspecto foi analisado o máximo déficit acumulado, característica relevante no caso de estudos de regularização e dimensionamento de reservatórios.

A figura 2 mostra a distribuição amostral do máximo déficit acumulado com indicação do valor do máximo déficit acumulado da série histórica com período 1931-1995. Observa-se que o valor desta série histórica (1931-1995) se apresenta estatisticamente indistinguível do conjunto das séries sintéticas geradas.

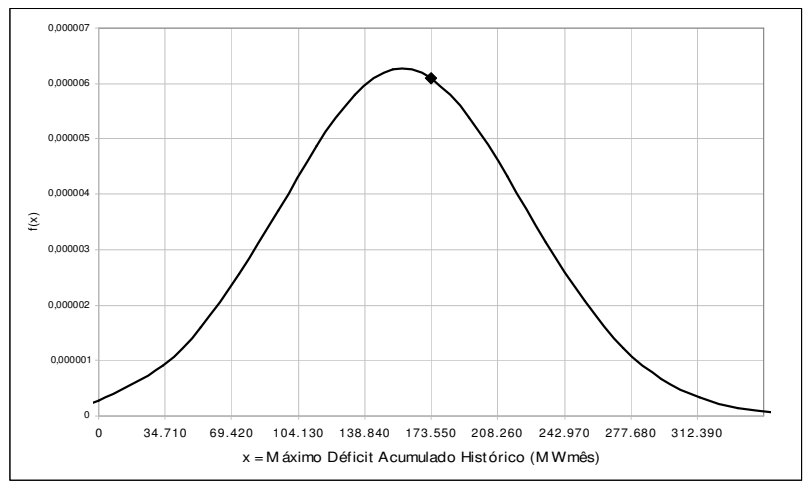

Figura 2 - Distribuição do máximo déficit acumulado mensal da série 1931-1995

\section{Determinação das Curvas de Regularização para Reservatórios Inicialmente Cheios}

Foram determinadas curvas de regularização tradicionais para vários valores do tempo de retorno utilizando as 1.000 séries sintéticas. Estas curvas foram comparadas com a curva de regularização da série histórica obtida pelo tradicional método de Rippl (Gomide, 1986). As curvas de regularização relacionam o volume útil (armazenamento máximo) de um reservatório com a vazão regularizada (energia garantida) para uma dada probabili- dade de sucesso expresso pelo tempo de retorno $\mathrm{Tr}$, e corresponde a condição inicial de reservatório cheio. Portanto indicam a energia que pode ser retirada de um reservatório de energia inicialmente cheio para que a probabilidade de sucesso em um período de " $n$ " anos seja:

$$
P\left(S_{n}\right)=(1-1 / \operatorname{Tr})^{n}
$$

Sucesso em $n$ anos, denotado por $S_{n}$, é definido como a não ocorrência de déficit em qualquer um dos $n$ anos. Por outro lado $s_{n}$ denota ausência de déficit no n-ésimo ano. Nota-se que a expressão (4) sabidamente válida para eventos independentes (Pinto et al., 1979) pode ser estendida para eventos dependentes, onde $T_{r}=1 / P_{r}\left(s_{n} \mid S_{n-1}\right)$ com $P_{r}\left(s_{n} \mid S_{n-1}\right)$ a probabilidade condicionada do sucesso no n-ésimo ano, dado que houve sucesso nos $n-1$ anos anteriores e suposta constante ao longo do tempo. Neste caso

$P_{r}\left(s_{n}\right)=P_{r}\left(s_{n-1} \cap s_{n}\right)=P_{r}\left(S_{n-1}\right) \cdot P_{r}\left(s_{n} \mid S_{n-1}\right)=\left[P_{r}\left(s_{n} \mid s_{n-1}\right)\right]^{n}$

e resulta a validade da expressão (4) também para eventos dependentes.

Para que as conclusões obtidas pela análise de um único reservatório de energia regularizando uma demanda igual a energia firme seja aplicável a um sistema complexo constituído por um conjunto de usinas e reservatórios hidrelétricos é necessário que:

a) O método da energia natural (Canambra, 1969) é aplicável;

b) A geração térmica é separável e seu valor pode ser considerado constante;

c) A confiabilidade é avaliada de forma estática, e mantida constante, e pode ser expressa pelo tempo de recorrência $\operatorname{Tr}=\frac{1}{1-(1-p)^{1 / n}}$, onde $p$ é a probabilidade de falha em $n$ anos (FILL e Groszewicz, 1989).

Em geral essas hipóteses são aceitáveis dentro da prática corrente do planejamento do setor elétrico no Brasil, constituindo inclusive a base dos métodos atualmente disponíveis para avaliação de riscos via teoria estocástica dos reservatórios (Fill e Groszewicz, 1987; 1989).

Inicialmente, para as 1.000 séries geradas, foram computadas as probabilidades de falha para 
diferentes volumes, para os 50 anos de extensão de cada série usando para tanto a aproximação da freqüência relativa:

$$
P\left[\text { falha } \mid S=D_{(j)}\right]=P\left[D>D_{(j)}\right]=\frac{j}{N+1}
$$

Sendo:

$S$ = armazenamento máximo do sistema;

$D_{(j)}=$ o j-ésimo máximo déficit acumulado ordenado das $N$ séries;

$N=$ número de séries.

Os máximos déficits acumulados para cada série gerada foram obtidos a partir do seguinte algoritmo:

$$
D(0)=0
$$

$$
\begin{aligned}
& D(t)=\operatorname{máx}\left\{\begin{array}{l}
D(t-1)+E_{f}-E(t) \\
0
\end{array}\right. \\
& D_{\text {máx }}=\operatorname{máx}[D(t)] \quad \text { para } t=1,2, \cdots n
\end{aligned}
$$

Onde:

$D_{\text {máx }}=$ máximo déficit acumulado,

$D(t)=$ déficit acumulado no mês $t$;

$E_{f}=$ demanda do sistema (energia firme);

$E(t)=$ energia afluente;

$n=$ extensão do período correspondente a cada série $(=50 \times 12=600)$.

Arbitrando-se valores para a demanda determinou-se o máximo déficit para cada série sintética mediante a aplicação do algoritmo descrito. Ordenaram-se os máximos déficits das várias séries sintéticas em ordem crescente. Para vários tempos de retorno selecionados calculou-se a probabilidade de sucesso correspondente a um horizonte de 50 anos mediante a expressão:

$$
P\left(S_{50}\right)=(1-1 / \operatorname{Tr})^{50}
$$

O máximo déficit acumulado correspondente ao tempo de retorno $\operatorname{Tr}$, $m a ́ x D_{T}$, será o j-ésimo valor da série dos máximos déficits acumulados ordenados máxD $(j)$ com:

$$
j=P\left(S_{50}\right) N=1000(1-1 / \operatorname{Tr})^{50}
$$

O valor de $\operatorname{máx} D_{(j)}$ corresponderá ao armazenamento necessário para regularizar a demanda $E_{f}$ com tempo de retorno $T r$. Repetindo-se o procedimento para diferentes valores de $E_{f}$ é possível traçar as curvas de regularização de $E_{f} \mathrm{em}$ função do armazenamento uma para cada $T r$.

\section{Determinação de Curvas de Regularização para Reservatórios Parcialmente Cheios e Confiabilidade Constante}

Para determinação de curvas de regularização para reservatórios parcialmente cheios, objeto deste estudo usou basicamente o mesmo método que a determinação das curvas de regularização convencionais, entretanto fixando-se os armazenamentos máximos e considerando diferentes valores iniciais para o armazenamento. Determinaram-se as respectivas demandas como função do armazenamento inicial de modo que $j$ séries sintéticas não apresentassem déficit (i.e. o reservatório nunca era totalmente deplecionado) e $N-j$ séries apresentassem déficit (i.e. armazenamento mínimo=0). $\mathrm{O}$ algoritmo usado foi o seguinte:

$S(0)=S_{\text {inicial }}$

$S(t)=\max \left\{\begin{array}{l}0 \\ \min \left\{\begin{array}{l}S(t-1)+E(t)-E_{f} \\ S_{\text {máx }}\end{array}\right.\end{array}\right.$

$$
\begin{aligned}
& \text { para } t=1,2, \cdots n \\
& S_{\text {min }}=\min [S(t)]
\end{aligned}
$$


Onde:

$S(t)=$ armazenamento no mês t;

$S_{\text {máx }}=$ armazenamento máximo;

$S_{\text {min }}=$ armazenamento mínimo;

$E(t)=$ energia afluente no mês t;

$E_{f}=$ demanda (energia firme);

$n=$ extensão do período da série em meses

Adotou-se para o armazenamento inicial $S(0) 20 \%, 40 \%, 60 \%$ e $80 \%$ do armazenamento máximo- $S_{\text {máx }}$.

Para cada um desses armazenamentos iniciais, as demandas foram determinadas por tentativas para que o número de séries com falhas $\left(S_{\min }=0\right)$ para cada tempo de recorrência fosse igual aos valores da tabela 3.

Aplicando-se o procedimento para cada $\mathrm{u} \mathrm{m}$ dos armazenamentos iniciais e diferentes tempos de retorno foram traçadas as curvas de regularização para o reservatório de energia parcialmente cheio.

\section{RESULTADOS}

Usando-se os parâmetros estatísticos da série com extensão de 1931 à 1995, obtiveram-se as curvas de regularização para reservatório equivalente inicialmente cheio conforme apresentado na figura 3 .

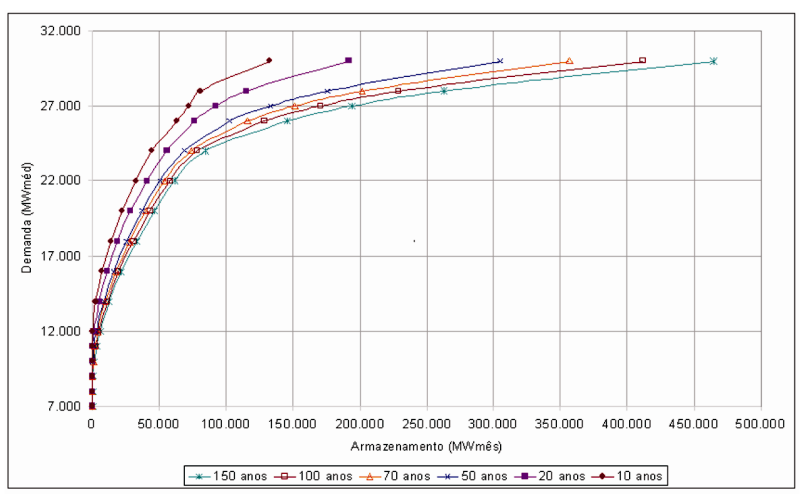

Figura 3 - Curvas de regularização para tempos de retorno selecionados (série 1931-1995)

Realizando uma comparação entra a curva de regularização histórica e as curvas para diversos tempos de retorno no caso da série 1931-1995 é possível concluir-se que a energia firme obtida da série histórica corresponde neste caso a um tempo de retorno da ordem de 100 anos.

As curvas de regularização para o reservatório equivalente parcialmente cheio para esse tempo de retorno $(\mathrm{Tr}=100$ anos) é mostrado na figura 4 .

Gomide (1986) em suas análises do sistema Sul-Sudeste encontrou um tempo de retorno da ordem de 60 anos para a energia firme obtida a partir da série histórica. Fill et al. (2003) encontraram para a mesma série histórica utilizada nesse artigo, valores para o tempo de retorno do sistema Sul-Sudeste próxima à de 100 anos.

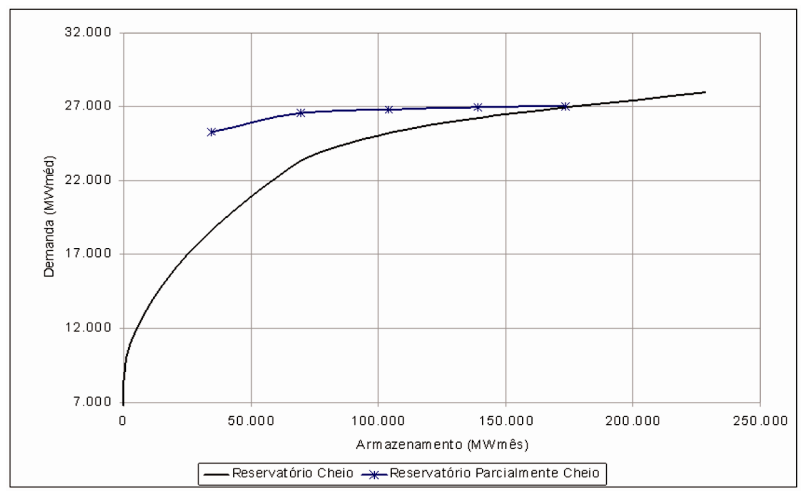

Figura 4 - Curva de regularização do reservatório parcialmente cheio com Tr de 100 anos e Smáx $=173.500$ MWmês (série 1931-1995)

Uma das hipóteses levantadas para a grande diferença entre os valores do tempo de retorno encontrados por Gomide (1986) e Fill et al. (2003) é que Gomide (1986) realizou as suas análises utilizando séries de afluências correspondentes à configuração de 1970 e uma série histórica de 1931 a 1970 , o que afeta sensivelmente a estimativa do coeficiente de autocorrelação, como já foi mencionado.

Por isso a análise cujos resultados são mostrados nas figuras 3 e 4 foi realizada também para os parâmetros da série com extensão de 1931 a 1980 e obtendo-se outras curvas de regularização para reservatórios cheios e parcialmente cheios.

A figura 5 apresenta para esse caso a curva de regularização para reservatórios cheios onde é possível comparar a curva de regularização histórica e as diversas curvas correspondentes ao tempo de retorno de 10, 20, 50, 70, 100 e 150 anos. 
Tabela 3 - Número de falhas em 1000 séries de 50 anos para diversos tempos de recorrência

\begin{tabular}{|c|c|c|}
\hline \multirow{2}{*}{ TEMPO DE RECORRÊNCIA } & \multicolumn{2}{|c|}{ " NÚMERO DE SÉRIES } \\
\hline & Com sucesso & Com falha \\
\hline 10 ANOS & 6 & 994 \\
\hline 50 ANOS & 364 & 636 \\
\hline 100 ANOS & 605 & 395 \\
\hline 150 ANOS & 716 & 284 \\
\hline
\end{tabular}

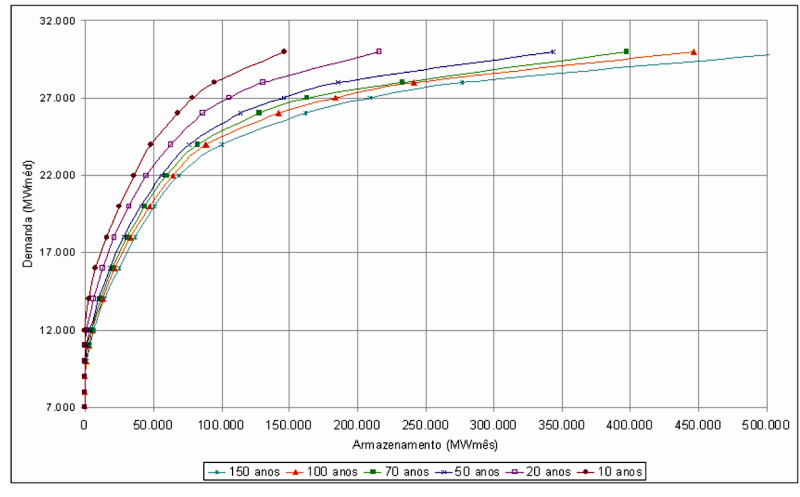

Figura 5 - Curvas de regularização para tempos de retorno selecionados (série 1931-1980)

Observa-se que a curva que mais se aproxima da série histórica é a curva com tempo de retorno de 70 anos. Este tempo de retorno é próximo aos valores encontrados por Gomide (1986), Fill (1989) e Fill e Groszewicz (1989) e indica que a série 19311980 aparentemente conduz a valores mais consistentes.

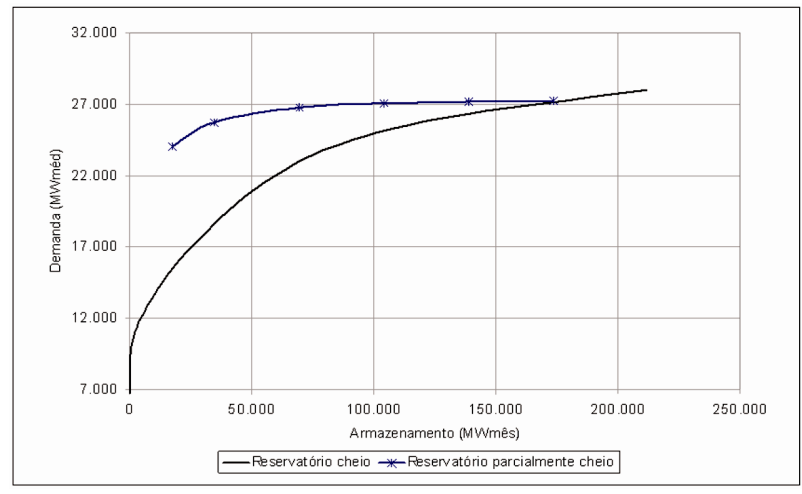

Figura 6 - Curva de regularização do reservatório parcialmente cheio com $T r$ de 70 anos e $S_{\text {máx }}=173.500$ MWmês (série 1931-1980)
Embora os resultados obtidos na análise da série 1931-1996 parecem menos consistentes a sua apresentação se justifica para induzir investigações futuras sobre o comportamento da autocorrelação das energias naturais do sistema sul-sudeste.

A figura 6 apresenta as curvas para reservatórios parcialmente cheios para $\operatorname{Tr}$ de 70 anos.

\section{CONCLUSÕES}

Este estudo teve como objetivo o desenvolvimento de um modelo de avaliação da energia garantida de um sistema hidrelétrico de geração, condicionado a um dado nível de armazenamento.

Para isto foi utilizado o método Monte Carlo com séries sintéticas de energias naturais geradas a partir dos parâmetros estatísticos da série histórica de afluências de energia do sistema Sul-Sudeste, configuração 2001.

Foram verificadas algumas inconsistências nos dados da série histórica original, o que levou a análise de dois cenários hidrológicos básicos, um com período de 65 anos (1931-1995), e outro com a série histórica reduzida de 50 anos (1931-1980).

Embora este artigo constitui-se em um estudo pioneiro e preliminar, permitem-se algumas conclusões que se acredita, sejam relevantes no equacionamento da operação de térmicas de emergência e da política de racionamento preventivo.

a) A formulação básica adotada para geração de séries sintéticas de energia natural afluentes (log-normal LN3, modelo autoregressivo $\mathrm{AR}(1)$ e desagregação através de cenários hidrológicos) mostrou-se adequada reproduzindo corretamente as variáveis re- 
levantes, particularmente o máximo déficit acumulado.

b) Sempre que o armazenamento se acha abaixo do máximo, a geração hidráulica deve ser reduzida para manter o risco futuro do déficit constante e igual ao risco associado à energia garantida do sistema.

c) Os resultados obtidos foram promissores, levando a crer que o método em princípio é adequado para definir uma política de operação de térmicas, requerendo, contudo ainda aperfeiçoamentos para a sua aplicação na prática.

d) A série histórica disponível de energias naturais aparentemente apresenta inconsistências que dificultam a estimativa correta dos seus parâmetros estatísticos, indispensáveis à geração de séries sintéticas.

Para dar continuidade a pesquisa apresentada neste artigo acredita-se que seja importante investigar com mais profundidade o comportamento anômalo das estimativas do coeficiente de autocorrelação e incorporar nos estimadores da redução da geração hidrelétrica cenários alternativos das afluências futuras. Além disso, uma abordagem ao problema via teoria estocástica dos reservatórios parece interessante pelo menos academicamente. No caso da aplicação do método à operação real do sistema interligado deve-se ainda considerar a representação individualizada das usinas e limitações do sistema de transmissão para determinar a distribuição da geração hidráulica entre as várias usinas do sistema.

\section{REFERÊNCIAS}

CANAMBRA ENGINEERING CONSULTANTS. Power study of South Brazil. App 17. Curitiba, 1969.

CENTRO DE HIDRÁULICA E HIDROLOGIA PROF. PARIGOT DE SOUZA - CEHPAR. Modelo simplificado de avaliação de risco - MSAR, Comparação com um modelo de simulação com séries sintéticas a subsistemas equivalentes (MSSSE). Curitiba: v.2, 1987. Projeto HG-60 - Consultoria em estudos energéticos e PCH.

FILL, H. D. O Método da energia natural como técnica de simulação em estudos energéticos. Revista Técnica do Instituto de Engenharia do Paraná, Curitiba, v. 20, p. 38-44. 1980.

FILL, H. D.; GROSZEWICZ, R. C. Validação de um modelo simplificado de avaliação de risco. In: SIMPÓSIO BRASILEIRO DE RECURSOS HÍDRICOS, 7., Sal- vador, BA. Anais... Salvador: ABRH, 1987. p. 354369.

FILL, H. D. Avaliação analítica da energia garantida incremental de uma usina hidrelétrica. In: SIMPÓSIO BRASILEIRO DE RECURSOS HÍDRICOS, 8., Foz do Iguaçu, PR. Anais... Foz do Iguaçu: ABRH, 1989.

FILL, H. D.; GROSZEWICZ, R. C. Distribuição do déficit em sistemas hidrelétricos com racionamento preventivo. In: SIMPÓSIO BRASILEIRO DE RECURSOS HÍDRICOS, 8., Foz do Iguaçu, PR. Anais... Foz do Iguaçu: $A B R H, 1989$. v. 1, p. 108-122.

FILL, H. D.; KAVISKI, E.; MINE, M. R. M.; CHELLA, M. R.; FREITAS, C. Projeto Energia: Metodologia para avaliação da contribuição energética de pequenas centrais hidrelétricas a fio d'água. Curitiba: UFPR LACTEC / CNPq, 2003. 58 p. Relatório Final.

GOMIDE, F. L. S. Teoria estocástica dos reservatórios aplicada ao planejamento energético de sistemas hidrelétricos. Curitiba, 1986. Paginação irregular. Tese (Titular), Departamento de Hidráulica e Saneamento, Universidade Federal do Paraná.

GOMIDE, F. L. S. Reunião realizada no CEHPAR. Curitiba, 2002. Citado por FILL, O. A. D. Comunicação pessoal. Curitiba, 2002.

GROSZEWICZ, R. C.; KAVISKI, E.; ILLICH, I.; MACHADO, J. F. P. Avaliação de três métodos de desagregação de afluências. In: SIMPÓSIO BRASILEIRO DE RECURSOS HÍDRICOS. 9., Rio de Janeiro, RJ. Anais... Rio de Janeiro: ABRH, 1991. p. 437-446.

HASHIMOTO, T.; STEDINGER, J. R.; LOUCKS, D. P. Reliability, resiliency and vulnerability criteria for water resource system performance evaluation, Water Resources Research, v. 8, n. 1, p. 14-20, 1982.

KAVISKI, E. Configuração. [comunicação pessoal]. Mensagem recebida por: <marcia.dhs@ufpr.br> em: 21 nov. 2002.

KELMAN, J.; PEREIRA, M. V. F. Critérios de avaliação para modelos de séries hidrológicas. In: SEMINÁRIO NACIONAL DE PRODUÇÃO DE TRANSMISSÃO E ENERGIA ELÉTRICA, 4. Rio de Janeiro, RJ. Anais... Rio de Janeiro, 1977.

KELMAN, J. Modelos estocásticos no gerenciamento de recursos hídricos. In: Modelos para gerenciamento de recursos hídricos. São Paulo: Nobel / ABRH, 1987. v. 1, cap. 4, p. 326-424.

KENDALL, M. G. The Advanced Theory of Statistics, v. 1, cap. 9, Charles Griffin \& Co, London, 1952.

LOUCKS, D. P.; STEDINGER, J. R., HAITH, D. A. Water resource systems planning and analysis. New Jersey: Prentice-Hall, 1981.

PINTO, N.L. de; HOLTZ, A.C.T.; MARTINS, J.A.; GOMIDE, F.L.S. Hidrologia básica. São Paulo: Ed. Edgard Blücher. 1976. 278p. 
PRESS, W. et al. Numerical recipes in pascal: The art of scientific computing. Cambridge: University Press, 1989.

STEDINGER, J. R. Fitting Log Normal Distributions to Hydrologic Data, Water Resour. Res., vol. 16, no. 3, pp. 481-490, 1980.

STEDINGER, J. R.; TAYLOR, M. R. Synthetic streamflow generation 1. Model verification and validation. Water resources research, 1982.

\section{Regulating Curves for Partially Full Reservoirs With Constant Reliability}

\section{ABSTRACT}

This paper aims to establish the power supply capability of an all interconnected hydroelectric systems as a function of the initial state of energy storage. A reasonable supply policy would be to keep the risk of future energy shortage constant considering at any time the available regulating storage. Obviously, this implies reducing hydropower generation whenever the available storage is less than the maximum storage capacity. The loss of hydropower generation may be supplied by thermal stand-by plants or in the absence of this source, supply cuts should be imposed. This paper proposes a method to evaluate that reduction of hydropower supply using Monte Carlo simulations with synthetic streamflow series. The synthetic streamflow generation analyzed two distinct scenarios, the first one using statistics from the complete available historic energy series (1931-1995) and the second one using the statistics from a sub-series covering 50 years of records (1931-1980). The sub-series presented an autocorrelation coefficient which was believed to be more realistic because of its similarity with other previous power supply studies. Comparison of the results using the historic and synthetic energy series shows that the firm energy corresponds to a return period close to 100 years for the 1931-1995 case and to roughly 70 years for the 1931-1980 case. This study concludes that the evaluation of the supply reduction is feasible and that the method can be used as a basis for programming thermal operation and power shortage in the Brazilian hydrothermal system. The reduction of firm energy has also been analyzed simulating the system over the historic natural energy hydrograph series. In this case, the reduction depends not only on the initial state of the energy storage, but also on the year simulation began. This shows that in practice, the decision about the reduction of hydrogeneration should consider, if possible, besides the available storage, the future energy inflow scenarios.
Key-words: Monte Carlo method, synthetic energy series, natural energy method, regulating curve, rationing planning.. 